\title{
Manifestations of central retinal artery occlusion revealed by fundus fluorescein angiography are associated with the degree of visual loss
}

\author{
HONGXIA GONG ${ }^{1}$, QIUYING SONG ${ }^{2}$ and LANHUI WANG ${ }^{3}$ \\ ${ }^{1}$ Department of Traditional Chinese Medicine; ${ }^{2}$ Visual Function Inspection Section; ${ }^{3}$ Department of Ocular Fundus Disease, \\ Tianjin Eye Hospital, Tianjin Medical University, Heping, Tianjin 300000, P.R. China
}

Received October 22, 2014; Accepted October 8, 2015

DOI: $10.3892 /$ etm.2016.3175

\begin{abstract}
The aim of this study was to investigate the association between central visual impairment and the characteristics of fundus fluorescein angiography (FFA) in patients with central retinal artery occlusion (CRAO). A total of 63 patients were diagnosed with CRAO by FFA. The visual dysfunction was classified into severe, mild and light degrees. Tropicamide was administered for mydriasis. FFA examination was performed using Heidelberg retinal tomography. The associations of age, gender and disease course with CRAO type were analyzed. Three types of manifestations were identified by FFA in 63 eyes, including poor perfusion (18 cases), exudation (22 cases) and mixed types ( 23 cases) of CRAO. No significant difference was found in age $(\mathrm{F}=0.171, \mathrm{P}=0.844)$ and disease course $(\mathrm{F}=0.016, \mathrm{P}=0.984)$ among the three types of $\mathrm{CRAO}$. Similarly, no significant difference was found in gender among the three types of CRAO $\left(\chi^{2}=0.176, P=0.916\right)$. The damage to vision caused by the exudation type of CRAO was not as severe as that caused by the poor perfusion and mixed types of CRAO. The distributions of damage severity caused by the poor perfusion and mixed types of CRAO were similar. In conclusion, the FFA observations for CRAO can be classified into three types of manifestations. The damage to vision in patients with CRAO is likely to be associated with poor perfusion in the retinal artery rather than exudation affecting the retina or optic disc. The patterns of clinical manifestations are not associated with age, gender or disease course.
\end{abstract}

Correspondence to: Dr Lanhui Wang, Department of Ocular Fundus Disease, Tianjin Eye Hospital, Tianjin Medical University, 4 Gansu Road, Heping, Tianjin 300000, P.R. China

E-mail: guoxuming0111@163.com

Key words: central retinal artery occlusion, vision acuity, fundus fluorescein angiography

\section{Introduction}

Retinal artery occlusion is a common eye disease, which is classified into central retinal artery occlusion (CRAO), branch retinal artery occlusion and ciliary retinal artery occlusion. $\mathrm{CRAO}$ is the most commonly occurring type of retinal artery occlusion, and is a disease of the eye that leads to sudden, acute and painless sight loss with poor prognosis. The main causes of CRAO are arteriosclerosis, embolus and inflammation (1-4).

As part of the nervous system of the brain, the central retinal artery is sensitive to hypoxia. When occlusion of the retinal artery occurs, blood supplies to the inner retina are blocked, leading to ischemia and necrosis of the inner retina, or even acute ischemic necrosis of the nerve fiber and ganglion cell layers. In addition, lesions of varying severity may occur in the retinal inner nuclear and inner plexiform layers. Nutrition is provided to the retinal visual cell layer by the choriocapillaris formed by the ciliary artery. Therefore, retinal visual cells retain their integrity at the early stage of CRAO, but begin to shrink at the late stage. The ciliary retinal artery is crucial for the protection of vision. The effect of ciliary retinal artery occlusion on vision is dependent on the size of the macular area supplied by the artery (2).

CRAO can be easily diagnosed by fundoscopic examination, and timely treatment is crucial for the alleviation of CRAO (5-8). In the present study, the fundus fluorescein angiography (FFA) results of CRAO patients attending the Tianjin Eye Hospital (Heping, China) were retrospectively investigated. The association between FFA and central visual impairment was analyzed, and the results may provide a basis for the clinical treatment and prognosis of CRAO.

\section{Materials and methods}

Patients. This study included 63 patients (63 eyes) who were diagnosed with CRAO by fundoscopic examination and FFA at the Tianjin Eye Hospital (Heping, China) between September 2010 and March 2014, including 28 males and 35 females (aged 43-75 years, with an average of 56.51 years). The time period between the time point at which the patient was aware of a sudden deterioration of vision and admission to our hospital was 1-15 days, with an average of 6.07 days. Among these 
Table I. Comparison of patient gender, age and disease course among the three types of CRAO.

\begin{tabular}{lcccc}
\hline CRAO type & Male $(\mathrm{n}=28)$ & Female $(\mathrm{n}=35)$ & Age $($ years $)$ & Disease course (days) \\
\hline Poor perfusion & 7 & 11 & $56.67 \pm 9.18$ & $6.22 \pm 4.40$ \\
Exudation & 10 & 12 & $57.00 \pm 8.32$ & $6.05 \pm 4.03$ \\
Mixed & 11 & 12 & $58.17 \pm 9.06$ & $6.00 \pm 3.94$ \\
F-value & & 0.171 & 0.016 \\
P-value & & 0.844 & 0.984 \\
\hline
\end{tabular}

Age and gender values are presented as mean \pm standard deviation. CRAO, central retinal artery occlusion.
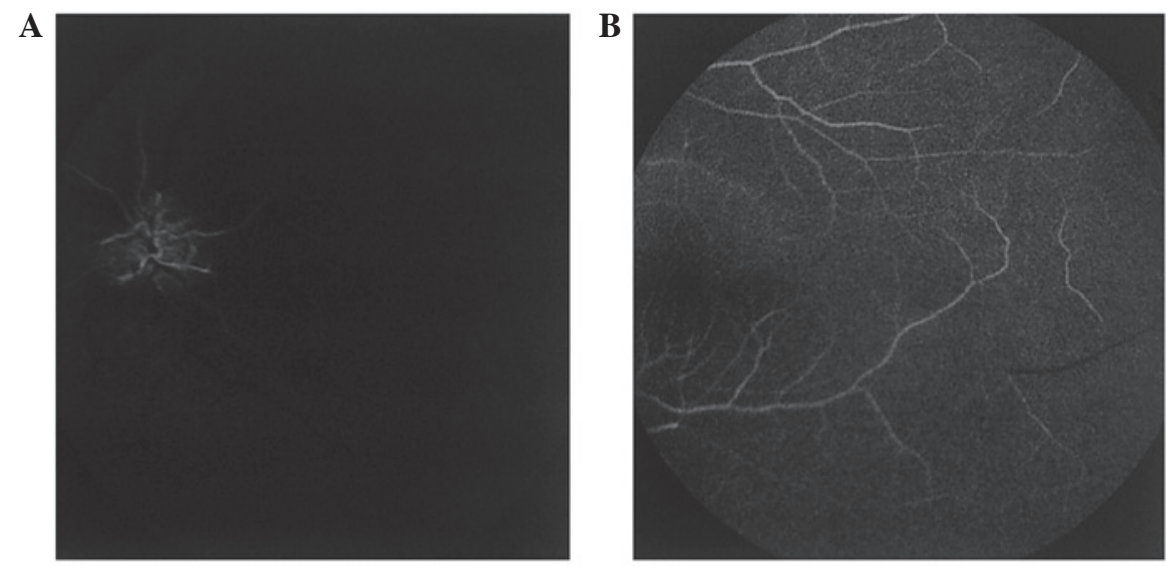

Figure 1. FFA of the poor perfusion type of CRAO at the (A) early and (B) late phases of FFA. At the early phase, poor perfusion in the retinal artery was observed (FFA time, $27.7 \mathrm{sec}$ ). At the late phase, perfusion was recovered in the peripheral retinal artery (FFA time, 12.45 min). The images were captured from the same patient. FFA, fundus fluorescein angiography; CRAO, central retinal artery occlusion.

patients, 44 cases had hypertension, 12 cases suffered from diabetes, and 9 cases had carotid stenosis or plaque. Exclusion criteria were: Ciliary retinal artery macula; heart rate outside 60-100 bpm and blood pressure outside 90-140/60-90 $\mathrm{mmHg}$, which are the allowed ranges for radiography; and history of trauma, surgery, oxygen inhalation or retrobulbar injection of drugs. All procedures were approved by the Ethics Committee of Nankai University (Tianjin, China). Informed consent was obtained from all patients or their families.

Central vision detection. Visual dysfunction is classified into severe, mild and light degrees (9). Patients with a severe degree of visual dysfunction cannot sense light or can only sense a hand movement in front of the eye; those with mild visual dysfunction can count the number of fingers or have a visual acuity of $0-0.08$; those with light visual dysfunction have a visual acuity $>0.1$.

FFA examination. Tropicamide was administered for mydriasis. FFA examination was performed using Heidelberg retinal tomography, with the observation time of radiography being 10-15 min (10). Sodium fluorescein (0.6 g; Zhongheng Pharmaceuticals, Guangzhou, China) was dissolved in $2 \mathrm{ml}$ sterile normal saline, and administered by intravenous infusion using a $22 \mathrm{G}$ needle within $5 \mathrm{sec}$. The starting time of central retinal artery perfusion and the time of the appearance of retinal vein laminar flow were recorded. The difference between the two time points was considered as the retinal arteriovenous phase.

Statistical analysis. All statistical analyses were performed using SPSS software, version 10.0 (SPSS, Inc., Chicago, IL, USA). The genders of the patients were analyzed using the $\chi^{2}$ test. Ages and the courses of disease were compared using analysis of variance (ANOVA). $\mathrm{P}<0.05$ was considered to indicate a statistically significant difference.

\section{Results}

Fundus performance evaluation for preliminary CRAO diagnosis. Prior to the diagnosis of CRAO, the fundus performance was evaluated. For the majority of the patients with CRAO, the retina exhibited a milky white haze, and the retinal artery was thinner than normal, with variable thickness. The color of the optic disc was lighter, with total or partial blur on the boundaries. The macular area showed 'cherry red' spots or edema. Segmental blood flow appeared in the retinal artery, or cotton-wool spots were observed in the retina. Five cases were found to be normal by fundoscopic examination. These results suggested that the evaluation of fundus performance was a useful step for preliminary CRAO diagnosis.

FFA indicates that there are three types of CRAO. In order to classify CRAO in each patient, FFA was performed. Among 
Table II. Severity of damage caused by the three types of CRAO.

\begin{tabular}{lccr}
\hline CRAO type & Severe damage, $\mathrm{n}(\%)$ & Mild damage, $\mathrm{n}(\%)$ & Light damage, $\mathrm{n}(\%)$ \\
\hline Poor perfusion & $4(22.22)$ & $8(44.44)$ & $6(33.33)$ \\
Exudation & 0 & $9(40.91)$ & $13(59.09)$ \\
Mixed & $4(17.39)$ & $11(47.83)$ & $8(34.78)$ \\
Total & $8(12.70)$ & $28(44.44)$ & $27(42.86)$ \\
\hline
\end{tabular}

CRAO, central retinal artery occlusion.
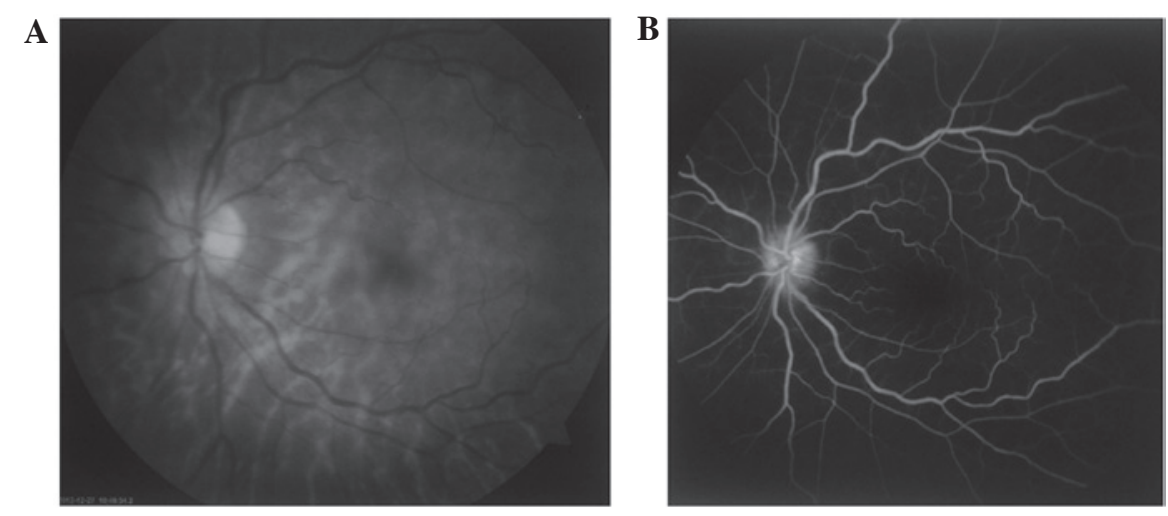

Figure 2. (A) Photograph of a fundus with the exudation type of CRAO. Edema was observed at the posterior pole of the retina. (B) FFA of the exudation type of CRAO at the late phase (FFA time, $11.27 \mathrm{~min}$ ). Fluorescence leakage by the retinal artery was displayed. Images were captured from the same patient. FFA, fundus fluorescein angiography; CRAO, central retinal artery occlusion.
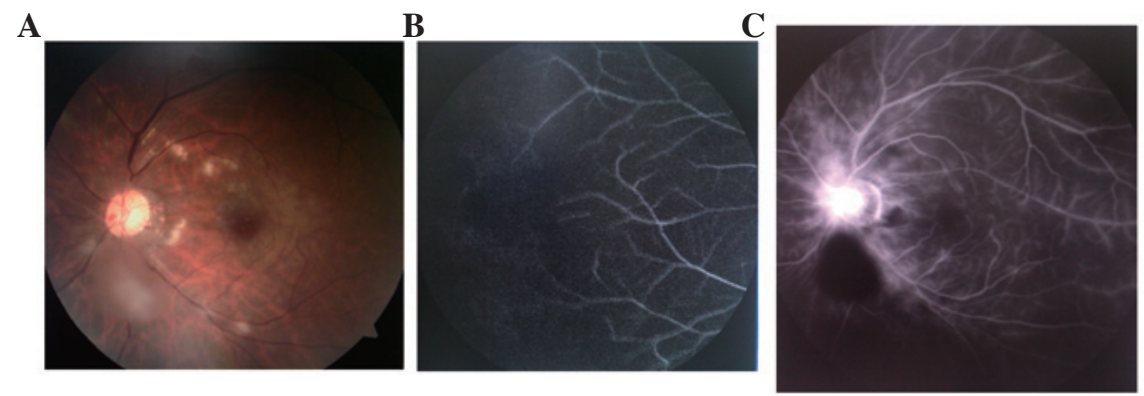

Figure 3. (A) Photograph of a fundus with the mixed type of CRAO. Edema and cotton-wool spots were observed on the retina. (B) FFA of the mixed type of CRAO at $29.2 \mathrm{sec}$. Non-perfusion in the peripheral retinal artery was shown. (C) FFA of mixed type of CRAO at 10.53 min. Fluorescence leakage of the retinal artery was observed. All images were captured from the same patient. FFA, fundus fluorescein angiography; CRAO, central retinal artery occlusion.

the 63 cases, 18 eyes were classified as the poor perfusion type, with a circulation time from the arm to the retina of $>23 \mathrm{sec}$. Arterial perfusion at the early phase of FFA was slow (Fig. 1A), but perfusion was recovered in the peripheral retinal artery at the late phase (Fig. 1B). The optic disc and vascular wall were normal or slightly stained. Moreover, 22 eyes were classified as having the exudation type of CRAO. These eyes had a normal retinal arteriovenous perfusion time. At the early phase, the optic disc exhibited high fluorescence with blurred boundaries (Fig. 2A). At the arteriovenous phase, the retinal vascular wall exhibited fluorescein leakage. At the late phase, fluorescein leakage occurred at the optic disc or retinal vascular wall (Fig. 2B). In addition, 23 eyes were considered as being affected by a mixed type of CRAO, having characteristics of the poor perfusion and exudation types (Fig. 3). The
FFA results within the study group identified three types of CRAO.

Gender and disease course do not correlate with the type of CRAO. Statistical analysis was performed to study the correlation of age, gender and disease course with different types of CRAO. Among the 63 eyes in the total study group, there were 18 eyes $(28.57 \%)$ of the poor perfusion type, comprising 7 eyes (11.11\%) from males and 1 eye (17.46\%) from a female. In the 22 eyes (34.92\%) of the exudation type, there were 10 eyes (15.87\%) from males and 12 eyes (19.05\%) from females. Among the 23 eyes (36.51\%) of the mixed type, there were 11 eyes (17.46\%) from males and 12 eyes (19.05\%) from females. The results identified no statistical difference in gender $\left(\chi^{2}=0.176, P=0.916\right)$ and disease course among the 
three types of CRAO ( $\mathrm{P}>0.05$; Table I). The data suggest that gender and disease course did not correlate with CRAO type.

Exudation type of CRAO causes less severe damage compared with poor perfusion or mixed types. To evaluate the severity of damage caused by the three types of CRAO, the numbers of patients with severe, mild and light degrees of CRAO were counted, respectively. Among the 63 eyes, 8 eyes $(12.70 \%)$ had severe damage, including 2 eyes with no ability to sense light, 3 eyes able to sense light and 3 eyes able to sense hand movement; 28 eyes (44.44\%) had mild damage, including 12 eyes with the ability to count fingers, and 16 eyes with a visual acuity of $0.01-0.08 ; 27$ eyes $(42.86 \%)$ had light damage. Among the patients having the poor perfusion type or mixed type of CRAO, 4 patients from each group had severe damage, and the percentages of patients having mild and light damage were similar in these two groups. By contrast, no severe visual damage was observed for patients with the exudation type of CRAO, with more than half of the patients having only light damage (Table II). These data demonstrate that the exudation type of CRAO caused less severe damage compared with the poor perfusion and mixed types.

\section{Discussion}

The average age of the 63 patients in this study was 56.51 years, which is lower than the average age (69.5 years) reported for patients with CRAO by researchers conducting a study in France (5). No statistically significant difference was observed in the incidence of CRAO between male and female patients, which differs from the results obtained in a Korean study, in which the CRAO incidence for males was higher than that for females (11). The typical symptom of CRAO was unrelieved amaurosis of the affected eye. Some patients experienced transient amaurosis. Fundoscopic examination showed that at the early stage of CRAO, the patients exhibited light retinal edema, retinal artery stenosis and unobvious changes of the optic disc, easily leading to an erroneous diagnosis that delayed treatment. The retinas of most eyes affected by CRAO appeared grey in color, which was even evident at the posterior pole. The macular area exhibited red lesions, which are known as 'cherry red spots'. The optic disc appeared light in color with tissue edema. In some eyes affected by CRAO, the retinal edema regressed, with the color returning to normal. At this time, fundoscopic examination showed normal results, but retinal tissue necrosis had already occurred, resulting in the retinal artery becoming thinner and occluded, and the optic nerve becoming atrophied; visual function is not able to recover from such damage (12).

FFA is a commonly used method for the diagnosis of CRAO. In contrast to fundoscopic examination, FFA images vary considerably, due to differences in the degree of artery occlusion and in the length of disease history. Among the 63 cases in the present study, 41 cases $(66 \%)$ had a longer arterial perfusion time than normal, of which 24 cases were relieved and 17 cases formed blurs in the peripheral and retinal macular regions at the late phase of FFA, revealed as non-perfusion fluorescence. In addition, 45 cases had hyperfluorescence leakage from the optic disc at an early phase of FFA, or fluorescence leakage from the retinal vascular wall at the arteriovenous or late phases. Comparison of the damage to vision for the three types of CRAO indicated that the poor perfusion and mixed types of CRAO caused more severe visual damage to vision than did the exudation type. The poor perfusion type had a similar distribution of damage severity as the mixed type. These observations concur with the findings of Altmann et al (13), indicating that poor perfusion of the retina is an important reason for visual dysfunction and the low treatment response.

Notably, it was observed that some of the patients with CRAO had normal fundoscopic examination and FFA results but severely damaged vision. This might be due to the formation of emboli from platelets, fibrin and cholesterol, which were quickly degraded and transported to the periphery. Therefore, it would not be possible to observe a totally occluded retinal artery by FFA, and irreversible lesions could have already occurred on ganglionic cells that were more sensitive to ischemia (14), leading to severely damaged vision. Because of this, it is important to not neglecting CRAO when patients present with symptoms of transient amaurosis. In addition, retinal neovascularization was not found in any of the CRAO cases. However, iris neovascularization was observed in 4 patients. We hypothesize that retinal neovascularization rarely occurs in CRAO because the epithelial layer of the retinal nerve is extensively damaged and the non-perfused region cannot generate. By contrast, iris neovascularization is caused by ischemia of the ophthalmic artery outside the retina. Previous studies have indicated that iris neovascularization usually occurs at 2-16 weeks after CRAO (15), and patients with secondary neovascular glaucoma account for $15 \%$ of all CRAO patients (16).

In conclusion, the FFA observations of CRAO can be classified into three types of manifestations. Visual damage in patients with CRAO is likely to be associated with poor perfusion in the retinal artery rather than with exudation of the retina or optical disc. The type of clinical manifestations does not correlate with age, gender or disease course.

\section{Acknowledgements}

This study was supported by Tianjin Eye Hospital and Nankai University.

\section{References}

1. Varma DD, Cugati S, Lee AW and Chen CS: A review of central retinal artery occlusion: Clinical presentation and management. Eye (Lond) 27: 688-697, 2013.

2. Hayreh SS: Acute retinal arterial occlusive disorders. Prog Retin Eye Res 30: 359-394, 2011.

3 . Hayreh SS: Ocular vascular occlusive disorders: Natural history of visual outcome. Prog Retin Eye Res 41: 1-25, 2014.

4. Hayreh SS, Podhajsky PA and Zimmerman MB: Branch retinal artery occlusion: Natural history of visual outcome. Ophthalmology 116: 1188-94.e1-e4, 2009.

5. Coisy S, Leruez S, Ebran JM, Pisella PJ, Milea D and Arsene S Systemic conditions associated with central and branch retinal artery occlusions. J Fr Ophtalmol 36: 748-757, 2013 (In French).

6. Padrón-Pérez N, Aronés JR, Muñoz S, Arias-Barquet L and Arruga J: Sequential bilateral retinal artery occlusion. Clin Ophthalmol 8: 733-738, 2014

7. Mößner A, Jurisch D and Meier P: Bilateral retinal artery occlusion. Ophthalmologe 111: 970-972, 2014 (In German).

8. McLeod D: Central retinal artery occlusion and cerebral stroke. Eye (Lond) 27: 1422, 2013. 
9. Wang RS, Lv PL, Wang B, Wan YQ and Lei XQ: Relationship between retinal circulation time and visual loss in patients with central retinal artery occlusion. Zhonghua Yan Di Bing Za Zhi 23: 177-179, 2007 (In Chinese).

10. Maguire JI and Federman JL: Intravenous fluorescein angiography. In: Duane's Ophthalmology. Tasman W and Jaeger EA (eds). 2013 edition. Lippincott Williams and Wilkins, Philadelphia, PA, 2013.

11. Park SJ, Choi NK, Seo KH, Park KH and Woo SJ: Nationwide incidence of clinically diagnosed central retinal artery occlusion in Korea, 2008 to 2011. Ophthalmology 121: 1933-1938, 2014.

12. Weger M, Pichler T, Franke GH, Haas A, Thaler HV, Kraigher-Krainer N, Groselj-Strele A, Wedrich A and Rabensteiner DF: Assessment of vision-related quality of life in patients with central retinal artery occlusion. Retina 34: 539-545, 2014.
13. Altmann M, Ertl M, Helbig H, Schömig B, Bogdahn U, Gamulescu MA and Schlachetzki F: Low endogenous recanalization in embolic central retinal artery occlusion-the retrobulbar 'spot sign'. J Neuroimaging 25: 251-256, 2014.

14. Miyake $Y$ (ed): Acquired Retinal Diseases. In: Electrodiagnosis of Retinal Disease. Springer, Tokyo, pp181, 2006.

15. Rudkin AK, Lee AW and Chen CS: Ocular neovascularization following central retinal artery occlusion: Prevalence and timing of onset. Eur J Ophthalmol 20: 1042-1046.

16. Körner-Stiefbold U: Central retinal artery occlusion-etiology, clinical picture, therapeutic possibilities. Ther Umsch 58: 36-40, 2001 (In German). 